The President summarized the Commission report for the triennium $1982-85$ and thanked members of the Organizing Committee for their cooperation and help in preparing the report. The President said that a few important fields had been left out in the report for lack of space. He mentioned in particular Intergalactic Medium and hoped that in the next report it would be possible to include this field. The report had already been mailed to all members of the Commission. Two circulars had also been sent out to all members of the Commission during this period which summarized the Commission activities. The members expressed their appreciation for being kept well informed.

Membership and Structure

The President remarked that Commission 34 is one of the largest of the Union with 470 existing members. During this triennium another 90 have expressed their desire to join the Commission and are going to be accepted at the end of this General Assembly; on the other hand, there have been only four resignations.

The President felt that considering the large size of the Commission and the diversity of subjects under its scope, one or more Working Groups could be formed in addition to the WG on nomenclature. A preliminary discussion on the structure of the Commission took place and as a consequence of it, a committee under the chairmanship of J. Lequeux and consisting of J.S. Mathis, P.A. Shaver, Y. Terzian and P. G. Wannier was formed to study this problem. It was also suggested that the Commission should take an active interest in the field of intergalactic medium.

The Committee on the structure of the Commission met after the business meeting and decided as a first step to form a Working Group on Planetary Nebulae and PN precursors, the chairman will be Y. Terzian. The Committee will report on his work at the next General Assembly.

\title{
Organizing Committee
}

The President then drew up the slate of officers and members of the organizing Committee for 1985-1988. Based on recommendations that he had received from members and past officers of the Commission, he proposed J.S. Mathis as the new Vice-President and K. de Boer, D.R. Flower, H.J. Habing, B.M. Shustov and D.G. York as new members of the $O C$. As has been the convention, the present Vice-President J. Lequeux will take over as President at the end of the XIX General Assembly. The continuing members of the $O C$ are: S. D'Odorico, B.G. Elmegreen, P.A. Shaver, P.G. Wannier and $M$. Peimbert. The proposed slate of officers and $O C$ members was approved by the Assembly. E.B. Kostyakova, U. Mebold, Y. Terzian and V. Radhakrishnan will retire from the $O C$ at the end of the current General Assembly. 
Symposia, Colloquia and Scientifc Sessions

Commission 34 sponsored two IAU symposia and cosponsored one colloquium just prior and after the XIX General Assembly. The President briefly commented upon the highly successful symposium on Star Forming Regions held in Tokyo earlier in the month,as well as on the colloquium on Hydrogen Deficient Stars and Related objects held in Mysore, during the same time. In addition, the Commission has cosponsored two Joint Discussions (Evolution in Young Populations in Galaxies and Supernovae) and four Commission meetings on important recent results during the XIX General Assembly. Brief reports on the Commission meetings are given below while the procedings of the Joint Discussions will be published in Highlights of Astronomy Volume 7 .

The future symposia sponsored and cosponsored by the Commission and already approved by the Executive Committee are Symposium 122 on Circumstellar Matter, to be held in Heidelberg in June 23-27 and Symposium 131 on Planetary Nebulae, to be held in Mexico City in late 1987. The President mentioned two other meetings which may be of interest to members of the Commission: the meeting on Interstellar Processes in Wyoming, USA in July 2-8 1986, and the one on Interaction of Supernova Remnants with the Interstellar Medium in Canada in June 1987.

\section{Working Group on ISM Nomenclature}

The activities of the Working Group were reported by its chairperson, Dr. Hélene Dickel. A preliminary manuscript describing a scheme for the "Designation and Nomenclature for Diffuse Radiating Sources" resulted from the deliberations of the WG and was circulated at the meeting. A list of resolutions concerning designations was presented and briefly explained. The publication of the report and the continuation of the WG was approved and the members of the WG were thanked for their efforts to clarify the nomenclature situation.

The resolutions of the WG on Nomenclature of Commission 34 and similar ones from Commission 40 (radio astronomy) endorsed by Commission 48 (high energy astrophysics) were coordinated by Commission 5 (documentation and astronomical data). The resulting recommendations for source designations from Commission 5 will be published in the 1986 revision of the IAU Style Book. Editors of astronomical Journals will be urged to include written requests to both authors and referees to insure that designation of astronomical sources are well documented and conform to the IAU rules.

\section{other Matters}

The President then asked Y. Terzian to report on the plans for a new catalog on planetary nebulae. Terzian mentioned the plans of Perek and Kohoutek to bring out an updated version of their Catalog on Galactic Planetary Nebulae. Furthermore, Mme. A. Acker of Strasbourgh Stellar Data Centre, has also been compiling a Catalog of Planetary Nebulae. Terzian described his efforts in convincing the authors to collaborate rather than duplicate the work; the Commission endorsed Dr. Terzian's endeavours on this matter. It is hoped that by the time of the next IAU Symposium on Planetary Nebulae in Mexico, the new catalog would be ready.

COMMISSION 34 MEETINGS

Dust

Chairperson: J.S. Mathis

21 November 1985 
Dr. D.C.B. Whittet (UK) summarized observations. Interstellar depletions of heavy elements (e.g., Ti) from the gas phase are well correlated with the average density of the interstellar medium, showing that they accrete onto grains. The only spectral feature in the entire $0.1-0.3 \mu \mathrm{m}$ wavelength range is the $0.22 \mu \mathrm{m}$ "bump"; this places some constraints on candidate materials for grains because many substances have strong absorptions which are not observed. The diffuse interstellar bands and the optical continuous extinction are not correlated with any ultraviolet extinction properties. The $9.7 \mu \mathrm{m}$ and $18 \mu \mathrm{m}$ (amorphous silicates) are seen in both emfssion and absorption. The 3.3 and $3.4 \mu \mathrm{m}$ absorption features are due to the $\mathrm{C}-\mathrm{H}$ stretch, but it is not yet possible to pin down the specific molecule. For cold clouds, the $3.07 \mu \mathrm{m}$ feature is fitted we11 by a mixture of water and ammonia ices, the $4.67 \mu \mathrm{m}$ band is $\mathrm{CO}$, and the $4.62 \mu \mathrm{m}$ is $\mathrm{CN}$.

Dr. Peter Martin (Canada) summarized several current theories of grains in general terms. The only unanimous conclusion is that amorphous silicates are present and that there is a large range in particle sizes. Several suggestions have been made for the origin of the $0.22 \mu \mathrm{m}$ bump: $\mathrm{OH}^{-}$ions on the surfaces of silicate grains (Duley); very small graphite (Greenberg); a range of graphites (Mathis "MRN"); hydrogenated amorphous carbon. The visual extinction is usually attributed to more than one substance, such as silicates and amorphous carbon.

Dr. Bruce Draine (USA) discussed formation and destruction of grains. Grains form in the atmospheres of both carbon and oxygen-rich stars, but it seems impossible to understand their formation in the best-studied star ( $\alpha$ Ori) on the basis of a steady state. If formation is episodic, present theory cannot make realistic predictions. Destruction caused by both sputtering and grain-grain collisions in supernova shocks should deplete grains in a time scale of $3 \times 10^{8}$ yrs if there is no formation within the interstellar medium itself. If there are many small grains, their total area is large enough to allow appreciable coagulation and accretion on this time scale. Thus, the grain size distribution in the interstellar medium must be heavily modified through growth processes as well as by shattering.

New Results on Planetary Nebulae

Chairperson: Y. Terzian

25 November 1985

R. Gathier reported on individual distances of several planetary nebulae. He showed that the positions of the PN central stars in the HR diagram indicate discrepancies with theory, concerning evolutionary timescales, and range of central star luminosities. D. Schönberner discussed a correlation between the absolute magnitude of central stars and the excitation of their nebulae. $P$. Wannier and $R$. Sahai presented observations of oxygen isotopic abundances from circumstellar envelopes of giant stars. ${ }^{17} \mathrm{O}$ is found to be overabundant in all the cases investigated. B. Zuckerman reported that he used the UKIRT telescope together with I. Gatley to observe $\mathrm{H}_{2}$ molecules from bi-polar PN, including the Dumbbell and NGC 2346. Y. Terzian presented $\lambda 6 \mathrm{~cm}$ VLA maps of several PN performed in collaboration with $\mathrm{C}$. Bignel1 and J. van Gorkom. Several of these objects were observed with an angular resolution of 0.4 ". These observations will be used with future ones to determine nebular expansions and distances. H.C. Bhatt and D.C.V. Mallik reported on the two dense $\mathrm{PN} \mathrm{Cn} 1-1$ and $\mathrm{Ml}-2$. The high densities indicate young objects, but the central stars show stellar spectra of $\mathrm{F}$ and $\mathrm{G}$ types and from IRAS observations low luminosities have been found indicating discrepancies with stellar evolutionary tracks. S. Kwok presented a VLA survey of stellar PN at $\lambda 6 \mathrm{~cm}$ with high resolution, and indicated that most have been spatially resolved. Y. Terzian, J. Phillips and $\mathrm{H}$. Payne described a survey of $\mathrm{OH}$ in $\mathrm{PN}$ and indicated the detection of $1612 \mathrm{MHz} O \mathrm{OH}$ line in $\mathrm{Vg} 2-2$ and NGC 6302. They strongly emphasifed that at these high sensitivities oH confusion from the galactic plane is extremely important. H.C. Bhatt and S.R. Pottasch reported on dust temperatures in PN from IRAS data, 
and suggested that the dust in PN may mainly be carbon dominated. Two papers were read in absentia. One by $E$. Kostyakova on the variability of IC 4997; and one by C.T. Hua on monochromatic images of small compact nebulae.

\section{Important Recent Results I}

Chairperson: J. Lequeux

25 November 1985

The following contributed papers were presented:

Observations of Nova RS Oph - R. Davis.

Optical Spectrum of RS Oph - T.P. Prabhu.

Theoretical Mode1 of RS Oph - F.D. Kahn.

2D Spectroscopy of Planetary Nebulae with a CCD Echelle - C.D. Mc Keith.

Molecular Astronomy in China - Xing Jun.

Models of Molecular Clouds - W. Boland.

Diffuse Bands and Polarization - W.B. Somerville.

Chemical Composition of NGC 2363 - S. Torres-Peimbert, M. Peimbert and M. Peña.

Important Recent Results II

Chairperson: F.D. Kahn

25 November 1985

The following contributed papers were presented:

On the Nebular N/O abundance ratio - R. Rubin.

Abundances in Liners - L. Binette.

Ionization in the Galactic Halo - J.N. Bregman and P.J. Harrington.

ISM work at Nançay - I. Kazes.

Very Low Frequency Recombination Lines - K.R. Anantaramaiah. Sa1ter.

Dust in H II Regions - M.A. Gordon, P.R. Jewe11, M. Kaftan-Kassim, and C.J.

BG $2107+49$, a Peculiar H II Region - L. Higgs. 